\title{
ANÁLISIS Y DIAGNÓSTICO DE LOS SISTEMAS DE PRODUCCIÓN OVINA EN EL SURESTE ESPAÑOL
}

\author{
ANALYSIS AND DIAGNOSIS OF SHEEP PRODUCTION SYSTEMS \\ IN SOUTHEAST SPANISH
}

\author{
Marín-Bernal, A.M. ${ }^{1}$ y Navarro-Ríos, M.J. ${ }^{1 *}$
}

¿Universidad Miguel Hernández. Departamento de Tecnología Agroalimentaria. Área de Producción Animal. Orihuela. Alicante. España. *mjnavarro@umh.es

\section{Palabras clave adicionales}

Caracterización. Desarrollo rural. Pequeños rumiantes.

\section{RESUMEN}

En el presente estudio se analiza la situación actual del sector ovino de carne en el espacio geográfico de la IGP (Indicación Geográfica Protegida) Cordero Segureño. Se presentan los resultados descriptivos de las 93 encuestas realizadas donde se analizan las principales fortalezas y debilidades que presenta la situación actual de los ganaderos. Se trata de una cabaña ganadera de alta rusticidad cuyo rendimiento productivo puede aumentar con una selección genética encaminada hacia una mayor pureza racial. Existe una buena predisposición a utilizar el sello de calidad, si bien el asociacionismo tanto en la producción como en la comercialización es bajo. El relevo generacional podría aumentarse si se logra mejorar a medio plazo el rendimiento productivo de las explotaciones con políticas de producción basadas en la calidad. Ternasco de Aragón, podría ser un buen referente cara a inspirar las medidas de mejora en la difusión de la nueva IGP Cordero Segureño.

\section{SUMMARY}

This study analyzes the current situation of the sheep meat industry in the geographical area of the IGP (Protected Geographical Indication) Cordero Segureño. We present the descriptive results of the 93 surveys that analyze the strengths and weaknesses that presents the current situation of farmers. This is a high rusticity livestock whose yield can be increased with a genetic selection aimed toward greater racial purity. There is a

\author{
AdDitionAL KEYWORDS \\ Characterization. Rural development. Small \\ ruminant.
}

willingness to use the quality mark, although the associations both in the production and marketing is low. The generational change could be increased if it can improve the medium-term growth performance of farms with production policies based on quality. Ternasco de Aragón, could be a good reference face to inspire improvement measures for distributing the new IGP Cordero Segureño.

\section{INTRODUCCIÓN}

El ganado ovino ejerce un papel relevante en el medio rural español al permitir fijar población permanente en zonas agrarias desfavorecidas y contribuye a la preservación medioambiental, social y económica, junto al ganado caprino (Castel et al., 2010), fundamentalmente mediante razas autóctonas perfectamente adaptadas. En los últimos años, el sector atraviesa una situación difícil, provocando una reducción progresiva de su censo a causa del importante aumento de los costes de producción por la fuerte subida del precio de las materias primas para los piensos ${ }^{1}$, así como, la reduc-

${ }^{1}$ Es el caso de la soja, la fuente de proteína más importante para piensos, cuyo precio subio a causa de la decisión de la UE en mayo de 2012 de eliminar los beneficios arancelarios a Argentina, de donde procede gran parte de la soja consumida en la UE para alimentación de su ganado. 


\section{MARÍN-BERNAL YNAVARRO-RÍOS}

ción del consumo de carne de cordero agravada por la crisis actual.

Solamente si se mantiene dicha actividad en condiciones de producción atractivas y rentables para las futuras generaciones, se podrá conseguir frenar este debilitamiento del sector. Para aplicar cualquier medida de fomento y apoyo, es necesario realizar un diagnóstico de la situación del que se desprendan medidas para la mejora.

Se considera una estrategia comercial deseable en el sector, el uso de marcas de calidad diferenciada (Gaspar et al., 2011). Actualmente existen 7 Indicaciones Geográficas Protegidas (IGP) de carne de cordero reconocidas en España. La IGP Cordero Segureño ha comenzado en 2014 la comercialización de los primeros corderos. El objetivo de este estudio es realizar un diagnóstico de la realidad socio-económica del sector ganadero asentado en el territorio reconocido por el consejo regulador de la citada IGP Cordero Segureño para poder deducir medidas estratégicas de mejora una vez que se pongan en marcha.

\section{MATERIAL Y MÉTODOS}

Se realizaron 93 encuestas en las comarcas de Huéscar (Granada), Los Vélez (Almería), Noroeste (Murcia) y Sierra de Segura (Jaén), en la delimitación geográfica considerada dentro de la IGP Cordero Segureño mediante muestreo aleatorio estratificado proporcional (figura 1). Las encuestas realizadas suponen el 10,68\% de la población estudiada.

Han sido considerados dos estratos, el geográfico teniendo en cuenta 4 comarcas (Huéscar, Los Vélez, Noroeste y Sierra de Segura) y el tamaño del rebaño (100-300; 300-500; 500-1000 y más de 1000 ovejas adultas).

El censo utilizado en el caso de Murcia, ha sido el de la petición anual de prima ganadera para ovino-caprino del año 2006. En las comarcas de Huéscar (Granada) y Sierra de Segura (Jaén), el censo de las respectivas Asociaciones de Defensa Sanitaria Ganadera (ADSG). En la comarca de Los Vélez (Almería) el censo facilitado por la Oficina Comarcal Agraria (OCA) de Vélez Rubio.

Los datos recogidos en la encuesta son los relativos a: tamaño de rebaño, razas, mano de obra, producciones, manejo y aspectos económicos (tabla I), variables que en estudios previos similares han demostrado su mayor poder discriminante a la hora de desarrollar el análisis tipológico (ToroMujica et al., 2012).

El programa estadístico utilizado ha sido el SPSS versión 16.0.

\section{RESULTADOS}

\section{TAMAÑO DE REBAÑO}

El tamaño medio (tabla II) de las explotaciones encuestadas $(377,7$ ovejas y 10,7 machos) agrupadas según estratos (tabla III) presenta desviaciones típicas elevadas, lo que indica una gran variabilidad.

La mayoría de los ganaderos encuestados pertenecen al estrato de menor tamaño (100300 ovejas adultas), resultando éste el más frágil. Varios de los ganaderos encuestados afirmaron estar pensando en abandonar el sector debido a la baja rentabilidad.

La tasa media de reposición anual es del $14,01 \%$ (tabla II). Sólo un 25,8 \% aseguraron que estaban aumentando sus rebaños (tabla IV).

La reposición de las hembras es prácticamente endógena; el 93,5\% de los encuestados lo hacen con animales propios del rebaño (tabla IV). La reposición externa de machos es del $12,9 \%$, frente a la reposición externa de hembras que alcanza sólo el $6,5 \%$. Ninguno de los encuestados pedían prestados machos para la monta.

\section{RAZAS}

La raza predominante es la Segureña, pero en pocos casos puede asegurarse la pureza racial ya que tan sólo el $24,7 \%$ de los encuestados pertenecen a la Asociación 


\section{ANÁLISIS Y DIAGNÓSTICO DE LOS SISTEMAS DE PRODUCCIÓN OVINA}

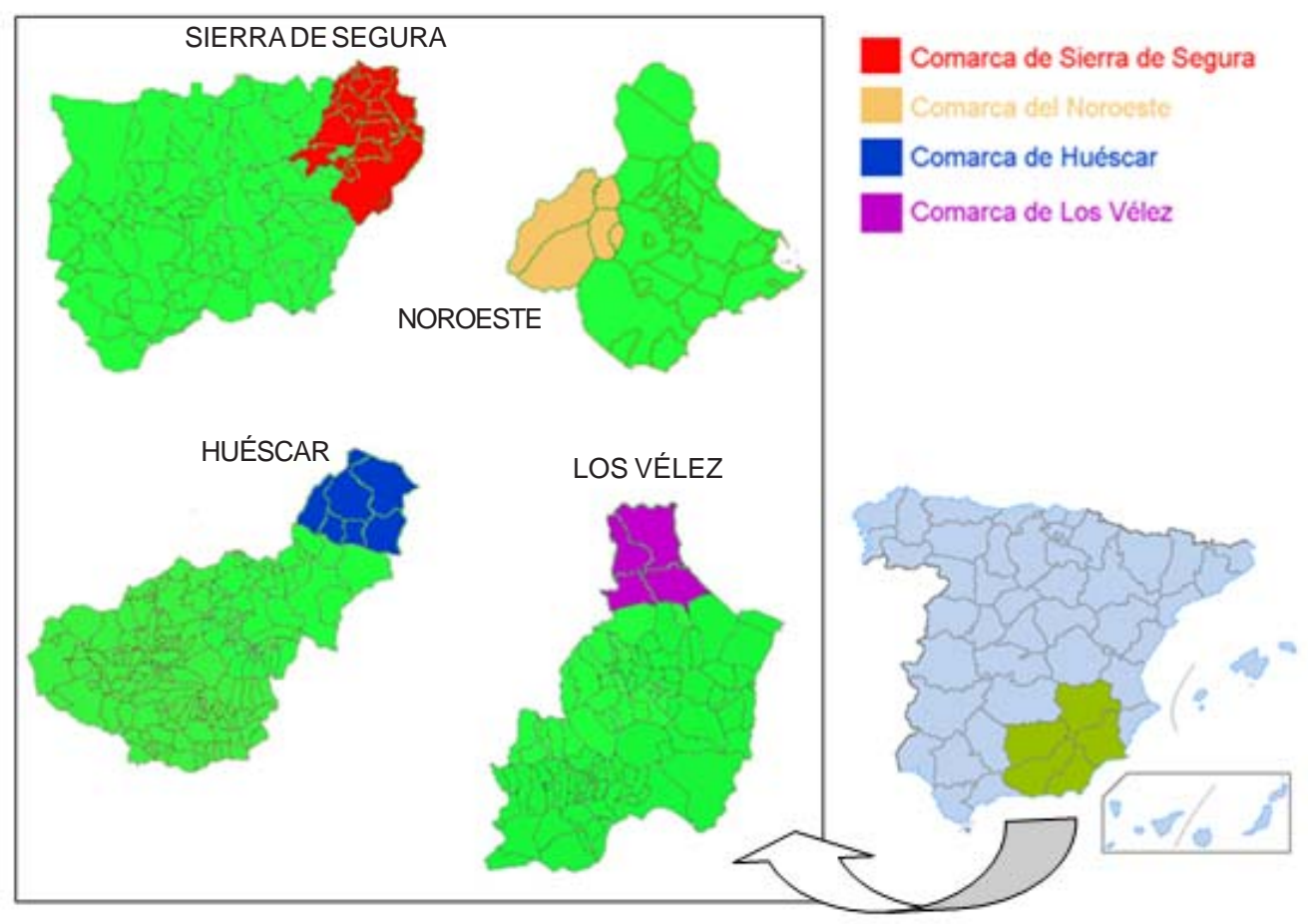

Figura 1. Comarcas donde se han realizado las encuestas. (Districts where surveys have been conducted).

Nacional de Criadores de Ovino Segureño (ANCOS) que es la que lleva el registro y control del libro genealógico de la raza ovina Segureña. Sólo un ganadero de los encuestados utilizaba un cruce industrial con Segureña y una raza foránea, la Romanov.

\section{Producciones}

Para todos los ganaderos encuestados el principal ingreso procede de la venta de corderos para carne. Además, algunos ganaderos vendían animales para la reproducción (fundamentalmente machos). Se trata de ganaderos socios de ANCOS con carta de progenitores y con garantía sanitaria.

Muy bajo porcentaje de ganaderos, el $9,7 \%$, pertenecen a algún tipo de cooperativa para la comercialización de los corderos (tabla IV), esta escasa tradición asocia- cionista dificultará sin duda la promoción en la zona del uso del sello de calidad garantizado de la IGP Cordero Segureño.

\section{MANODE OBRA}

La media de la mano de obra empleada (tabla V) por explotación es de 1,31 UTA (rango 0,41-3,11 UTA). Sólo un 9,6\% de los encuestados dispone de empleados fijos y un $15,1 \%$ contrata mano de obra asalariada de forma eventual (tabla IV). El 75,3\% de los ganaderos no emplean ningún tipo de mano de obra asalariada, lo que nos indica el marcado carácter familiar de este sector.

Las nueve explotaciones con mano de obra asalariada fija son las de mayor tamaño. Ocho de ellas son explotaciones de carne extensivas con un gran efectivo (86 UGM de media), dedicándose los emplea- 
MARÍN-BERNAL YNAVARRO-RÍOS

Tabla I. Variables cuantitativas y cualitativas. (Quantitative and qualitative variables).

\begin{tabular}{ll}
\hline Variables cuantitativas & Variables cualitativas \\
\hline Tamaño de rebaño & Evolución del tamaño de rebaño \\
Tasa de reposición & Procedencia de la reposición \\
Óvejas/macho & Razas presentes en las explotaciones \\
Mano de obra & Venta de los corderos \\
Edad mano de obra & Continuidad de la explotación \\
Edad del titular & Elaboración del pienso \\
Antigüedad en el oficio & Tipo de pastoreo \\
Ovejas/UTA total & Lactancia artificial \\
Edad destete (meses) & Trashumancia \\
Peso al destete & Ganaderos interesados en acogerse a IGP \\
Peso de venta & \\
Prolificidad & \\
Precio medio de los corderos & \\
Ingresos brutos anuales & \\
\hline
\end{tabular}

dos fijos básicamente a la tarea del pastoreo. La otra ganadería es una explotación de carne intensiva con gran número de ovinos (200 UGM). En este caso el personal asalariado fijo se encarga sobre todo de las tareas relacionadas con la alimentación de los animales y la paridera.

La edad media del titular es de 50 años (tabla V) con una relación de 287,1 ovejas/ UTA y la edad media de la mano de obra asalariada fija es de 43 años, con elevada variabilidad en ambas (tabla V). De las ganaderías con mano de obra asalariada fija, la media de empleados es de 1,11 UTA por

Tabla II. Tamaño medio de rebaño y tasa de reposición. (Average size of herd and restocking fee).

\begin{tabular}{lcccc}
\hline & N & Media & DT & Intervalo \\
\hline Ovejas & 93 & 377,7 & 311,62 & $100-2000$ \\
Machos & 93 & 10,7 & 8,7 & $1-48$ \\
UGM & 93 & 37,7 & 31,2 & $10-200$ \\
Ovejas/macho & 93 & 37,9 & 12,9 & $20-100$ \\
TR & 93 & 14,01 & 4,96 & $0-26,7$ \\
\hline
\end{tabular}

$\mathrm{DT}=$ desviación típica; $\mathrm{UGM}=$ unidades de ganado mayor; TR= tasa de reposición (\%). explotación (rango 1-2).

La media de mano de obra eventual en las explotaciones que disponen de este personal es de 0,22 UTA/explotación. Las tareas fundamentales que desempeña el personal asalariado eventual, son el pastoreo de las ovejas sin corderos y ayuda en la paridera.

Un $32 \%$ de los casos afirmaron tener asegurada la continuidad de la explotación, frente a un $12 \%$ que confirmaron el cierre de la misma tras su jubilación (tabla IV). El $56 \%$ restante no sabía si la explotación continuaría. Si analizamos la edad media de

Tabla III. Valores descriptivos de los rebaños según estratos y tamaño. (Descriptive values of herds as strata and size).

\begin{tabular}{lccc}
\hline $\begin{array}{l}\text { Estrato } \\
\text { (ovejas) }\end{array}$ & Ganaderías & $\begin{array}{c}\text { O } \\
\text { Media } \pm D T\end{array}$ & $\begin{array}{c}\text { O/M } \\
\text { Media } \pm D T\end{array}$ \\
\hline $100-299$ & $41(44,1)$ & $178,3 \pm 50,9$ & $36,3 \pm 13,2$ \\
$300-499$ & $31(33,3)$ & $348,3 \pm 46,5$ & $40,1 \pm 12,3$ \\
$500-1000$ & $17(18,3)$ & $647,8 \pm 111,1$ & $36,8 \pm 13,5$ \\
$>1000$ & $4(4,3)$ & $1502,5 \pm 450,9$ & $42,9 \pm 12,4$ \\
\hline
\end{tabular}

$\mathrm{O}=$ ovejas; $\mathrm{M}=$ macho; $\mathrm{DT}=$ desviación típica.

Archivos de zootecnia vol. 63, núm. 243, p. 522. 


\section{ANÁLISIS Y DIAGNÓSTICO DE LOS SISTEMAS DE PRODUCCIÓN OVINA}

Tabla IV. Variables cualitativas de los rebaños en el área de la IGP Cordero Segureño. (Qualitative variables of flocks in the Protected Geografical Indication Cordero Segureño).

\begin{tabular}{|c|c|c|c|}
\hline & Frecuencia & $\%$ & $\%$ acumulado \\
\hline \multicolumn{4}{|c|}{ Evolución del tamaño de los rebaños en los últimos 5 años } \\
\hline en aumento & 24 & 25,8 & 25,8 \\
\hline estable & 53 & 57 & 82,8 \\
\hline en retroceso & 16 & 17,2 & 100,0 \\
\hline \multicolumn{4}{|l|}{ Procedencia de los animales reproductores } \\
\hline reposición con hembras propias & 87 & 93,5 & - \\
\hline reposición con machos propios & 81 & 87,0 & - \\
\hline pide machos prestados para la monta & 0 & 0,0 & - \\
\hline \multicolumn{4}{|l|}{ Razas presentes en las explotaciones } \\
\hline Segureña (Pertenecen a ANCOS) & 23 & 24,7 & 24,7 \\
\hline Segureña con diversos grados de pureza & 69 & 74,2 & 98,9 \\
\hline cruce de Segureña con Romanov & 1 & 1,1 & 100,0 \\
\hline \multicolumn{4}{|l|}{ Vende los corderos } \\
\hline pertenece a cooperativa & 9 & 9,7 & 9,7 \\
\hline no pertenece a cooperativa & 84 & 90,3 & 100,0 \\
\hline \multicolumn{4}{|l|}{ Tipo de mano de obra } \\
\hline asalariada eventual & 14 & 15,1 & 15,1 \\
\hline asalariada fija & 9 & 9,6 & 24,7 \\
\hline solo familiar & 70 & 75,3 & 100,0 \\
\hline \multicolumn{4}{|l|}{ Continuidad de la explotación } \\
\hline no tiene & 11 & 12 & 12 \\
\hline hijo/a & 30 & 32 & 44 \\
\hline $\mathrm{ns} / \mathrm{nc}$ & 52 & 56 & 100,0 \\
\hline \multicolumn{4}{|l|}{ Tipo de alimentación en pesebre } \\
\hline elaboración propia más pienso compuesto & 2 & 2,2 & 2,2 \\
\hline elaboración propia más Unifeed & 1 & 1,1 & 3,3 \\
\hline Unifeed & 3 & 3,3 & 6,6 \\
\hline pienso compuesto & 4 & 4,4 & 11 \\
\hline elaboración propia & 83 & 89,2 & 100,0 \\
\hline \multicolumn{4}{|l|}{ Tipo de pastoreo } \\
\hline pastoreo conducido o guiado & 76 & 81,7 & 81,7 \\
\hline pastoreo en cercas & 11 & 11,8 & 93,5 \\
\hline mezcla de ambos & 6 & 6,5 & 100,0 \\
\hline \multicolumn{4}{|l|}{ Interesados en acogerse a IGP } \\
\hline interesados & 57 & 61,3 & 61,3 \\
\hline no interesados & 36 & 38,7 & 100,0 \\
\hline
\end{tabular}

estos últimos ganaderos, es inferior a la media del total de la muestra (46 años frente a 50). Se trata mayoritariamente de ganaderos con hijos menores de edad, que esperan o desean que sus hijos se dediquen a otra actividad, ya que consideran que la explotación conlleva mucho esfuerzo y dedicación personal, sin la compensación económica ni calidad de vida suficiente.

\section{MANEJo}

La mayoría de las explotaciones $(89,2 \%)$, bien en parte o bien en su totalidad, elaboran su propio pienso para las reproductoras, aunque sólo el $37 \%$ de las mismas cultivan sus propias materias primas (fundamental- 


\section{MARÍN-BERNAL YNAVARRO-RÍOS}

Tabla V. Mano de obra, mano de obra asalariada, edad del titular, número de ovejas/UTA total, tipo mano de obra, continuidad de la explotación, tipo de alimentación, precio medio de los corderos e ingresos brutos anuales. (Labor, hired labor, age, number of sheep owner/UTA total, type of labor, exploitation, continuity of supply type, average price of lambs and annual gross income).

\begin{tabular}{lccc}
\hline & $N$ & Mínimo-Máximo & Media \pm DT \\
\hline Mano de obra & & & \\
UTA total & 93 & $0,41-3,11$ & $1,31 \pm 0,53$ \\
UTA familiar & 93 & $0-3$ & $1,18 \pm 0,43$ \\
UTA asalariada fija & 93 & $0-2$ & $0,11 \pm 0,34$ \\
$\quad$ UTA asalariada eventual & 93 & $0-0,41$ & $0,04 \pm 0,09$ \\
Mano de obra asalariada & & & \\
$\quad$ Edad mano de obra fija & 9 & $25-64$ & $43 \pm 12,35$ \\
UTA asalariada fija & 9 & $1-2$ & $1,11 \pm 0,33$ \\
UTA asalariada eventual & 16 & $0,3-0,41$ & $0,22 \pm 0,11$ \\
Edad del titular y antigüedad en el oficio & & & \\
$\quad$ Edad del titular & 93 & $25-85$ & $50 \pm 12$ \\
$\quad$ Antigüedad en el oficio & 93 & $13-50$ & $20 \pm 13$ \\
№ ovejas/UTA total & & & \\
$\quad$ No ovejas/UTA total & 93 & $67-973$ & $287,1 \pm 167,4$ \\
Manejo corderos & & & \\
$\quad$ Edad destete (meses) & 81 & $1-3$ & $1,64 \pm 0,27$ \\
Peso al destete (kg) & 81 & $11-23$ & $14,45 \pm 2,03$ \\
$\quad$ Peso de venta (kg) & 93 & & $22,66 \pm 2,46$ \\
Precio medio de los corderos por comarca (euros) & & & \\
$\quad$ Huéscar & 46 & $40-60$ & $51,1 \pm 3,12$ \\
$\quad$ Los Vélez & 10 & $40-60$ & $49,5 \pm 3,18$ \\
$\quad$ Noroeste & 28 & $45-60$ & $53,3 \pm 7,27$ \\
$\quad$ Sierra de Segura & 9 & $45-54$ & $48,4 \pm 4,83$ \\
Ingresos brutos anuales por comarca (euros/ovejas) & & & \\
$\quad$ Huéscar & 46 & $56,3-112,7$ & $80,37 \pm 11,98$ \\
Los Vélez & 10 & $67,6-131,2$ & $84,94 \pm 20,26$ \\
$\quad$ Noroeste & 28 & $63,6-116,6$ & $90,89 \pm 11,47$ \\
$\quad$ Sierra de Segura & 9 & $62,7-106,5$ & $79,63 \pm 13,61$ \\
\hline
\end{tabular}

DT= Desviación típica.

mente cebada, obteniendo grano y paja). En un reducido $3,3 \%$ de los casos, los animales son alimentados con mezclas completas y en un solo caso combinan estas mezclas con su propio pienso (tabla IV).

El pastoreo, en su mayoría es conducido o guiado $(81,7 \%$ de los encuestados $)$, en el resto de los casos, el pastoreo se realiza en cercas o combinando ambas modalidades (cercas y pastoreo guiado) (tabla IV).

La trashumancia sólo la realizan 5 de los ganaderos encuestados $(5,4 \%)$ todos de la Comarca de Sierra de Segura (Jaén).

La edad media de destete de los corderos es de unos 50 días con un peso medio de $14,4 \mathrm{~kg}$ (tabla V). El peso medio de venta es de 22,6 kg (rango 15-26 kg).

La lactancia artificial de los corderos es prácticamente inexistente en este tipo de explotaciones pues tan sólo una de las explotaciones encuestadas afirmó llevarla a cabo. 


\section{ANÁLISIS Y DIAGNÓSTICO DE LOS SISTEMAS DE PRODUCCIÓN OVINA}

Tabla VI. Comparación de variables entre interesados y no interesados en formar parte de la IGP. (Comparison of variables between interested and uninterested in part by the IGP).

\begin{tabular}{lccccr}
\hline & N & Mín & Máx & Med & DT \\
\hline Edad del titular & & & & & \\
$\quad$ Interesados & 57 & 28 & 70 & 47,61 & 10,3 \\
$\quad$ No interesados & 36 & 25 & 85 & 54,33 & 13,8 \\
Tamaño medio rebaño & & & & & \\
$\quad$ Interesados & 57 & 100 & 2000 & 459 & 362 \\
$\quad$ No interesados & 36 & 100 & 600 & 248 & 129 \\
\hline
\end{tabular}

La prolificidad (media 1,2 corderos/oveja) estuvo correlacionada $(\mathrm{p}<0,01)$ con el tamaño del rebaño, de forma que a mayor tamaño de rebaño mayor prolificidad.

\section{AsPeCtOSECONÓMICOS}

El precio medio de los corderos (tabla $\mathbf{V}$ ) es de 51,3 euros registrándose el mínimo en Sierra de Segura (Jaén) con 48,4 euros.

Los ingresos brutos anuales medios de los ganaderos ascienden a 83,95 euros/oveja. Los ganaderos del Noroeste (Murcia) obtienen los mayores ingresos, 90,89 euros/ oveja y los de Sierra de Segura (Jaén) los menores $(79,63$ euros/oveja).

El 61,3\% de los ganaderos encuestados afirmaron estar interesados en acogerse a la IGP Cordero Segureño, fundamentalmente porque confían en que este distintivo de calidad puede reportarles algún beneficio económico por las ventajas para la comercialización de la carne (tabla IV).

La edad media del grupo que afirmó no estar interesado (tabla VI) es de 54 años con tamaño de rebaño de 249 cabezas, frente a tamaños medios de rebaño de 459 cabezas y una edad media de 47, en el caso de los interesados en acogerse a la IGP. Es decir, los titulares dispuestos a acogerse a la IGP son personas más jóvenes con mayor cabaña ganadera y mayor grado de profesionalización; el $31 \%$ de los interesados pertenecen a ANCOS y sólo el 13,88\% de los que no lo están. Además un $61 \%$ de los interesados pertenece también a algún otro tipo de asociación frente al $33 \%$ en el caso de los que no muestran interés por la IGP, lo que indica también una mayor predisposición al asociacionismo entre los interesados en acogerse al consejo regulador de la IGP Cordero Segureño.

\section{DISCUSIÓN}

En los últimos años se ha producido un cambio considerable en el tamaño de los rebaños de ovino de carne. Hace dos décadas, el tamaño medio en la provincia de Murcia era de 190 cabezas (Círia et al., 1991, citado por ${ }^{1}$ Poto et al., 2000). Actualmente, según este estudio, alcanza las 377,7 cabezas. También en Andalucía, según Sierra (2003), el número de cabezas de ganado ha experimentado un aumento significativo del tamaño medio, pasando de 275 en 1993 a 611 en 2003. En definitiva, la tendencia es hacia la desaparición de las pequeñas explotaciones, por jubilación o venta y al aumento de explotaciones de tamaño medio o elevado (más de 300 hembras reproductoras). Esta tendencia puede explicarse, entre otros aspectos, por la necesidad de adaptar la mano de obra existente a un tamaño rentable, así como la influencia de la regularización que la Organización Común de Mercados (OCM) establecía a la hora de regular la subvención de prima ganadera por cabeza de ganado.

La tasa media de reposición, de un $14,01 \%$, contrasta con la mayor tasa de

${ }^{1}$ Poto, A.; Lobera, J.B. y Peinado, B. 2000. Razas autóctonas de Murcia. Estimación del censo y aptitudes. Arch Zootec, 49: 107-114. 


\section{MARÍN-BERNAL YNAVARRO-RÍOS}

reposición encontrada en los estudios de García et al. (2005), de un $20 \%$, o el de Valerio et al. (2010) con una tasa del $46,8 \%$. Daza (2002) indica que la vida media de las ovejas reproductoras en España, oscilan entre 5-6 años, mientras que en este trabajo la vida útil de las hembras productoras es de 7,13 años, lo que indica un potencial genético de gran rusticidad y buenos resultados productivos.

En ningún caso se registra la petición de machos prestados para la monta, lo que contrasta con lo observado por Milán (1997) quien indica que es común el intercambio de machos entre ganaderos de ovinos de raza Ripollesa en Cataluña.

Existe escasa tradición asociacionista, aspecto negativo a la hora de intentar difundir el sello de calidad garantizado como es la IGP Cordero Segureño. Así lo constata también el estudio de Sierra (2003), en el que el cooperativismo en Andalucía arrojó una tasa de $8,3 \%$. Sin embargo, y según el mismo estudio, otras comunidades autónomas como Aragón y Castilla y León, los ganaderos asociados a algún tipo de cooperativa superaban el $80 \%$. Difundir el ejemplo de estas comunidades autónomas podría ser una medida de actuación para aumentar la escasa tendencia al cooperativismo murciano y andaluz.

La edad media del titular de las explotaciones estudiadas (50 años) es similar a la referida en otros estudios sobre sistemas ovinos en otras provincias españolas ( $\mathrm{Ga}$ llego et al., 1993; Mainar, 1995). En un trabajo realizado sobre la oveja Xisqueta en la provincia de Huesca y Lérida (Avellanet, 2006), la edad media de sus titulares, de 54 años es considerada crítica, afirmando que podrían desaparecer el $26,7 \%$ de las explotaciones sin relevo generacional. Este dato indica la importancia que tiene la puesta en marcha de acciones destinadas a promover entre los jóvenes el interés por dedicarse a esta actividad.

La productividad media de la mano de obra obtenida en este estudio, 287,1 ovejas/
UTA, supera a la media nacional obtenida en el estudio de De Rancourt et al. (2006), de 206 ovejas/UTA, o a la de las explotaciones ecológicas de ovino lechero en CastillaLa Mancha, con 217 ovejas/UTA, aunque al ser lecheras requieren más mano de obra (Toro-Mujica, 2011). Se trata de un indicador de peso en el análisis del resultado productivo total de la explotación, siendo en el caso del ovino de carne en régimen extensivo en zona de montaña un aspecto clave para alcanzar el máximo de eficiencia tanto de recursos humanos, como técnicos y medioambientales y supone un aspecto positivo cara a futuras acciones de mejora.

La incertidumbre en la continuidad de la explotación asciende a un $68 \%$. Se trata de un resultado que indica unas expectativas del sector altamente frágiles, aspecto común en sistemas de producción similares como es el caso del ovino de raza Alcarreña, en el que según el estudio de García et al. (2011a), esta inseguridad aumentaba a una proporción del 91,7 \% de los ganaderos encuestados.

El pastoreo en cercas se practica escasamente (11,8\% de los casos), comparado con otras zonas de Andalucía, donde alcanza un $42 \%$, o Extremadura, donde prácticamente la totalidad de los ganaderos encuestados (98\%), lo practicaba (Sierra, 2003). El caso opuesto se encuentra en Aragón, donde su uso es prácticamente testimonial (3\%) (Sierra, 2003). En este último estudio se afirma que el pastoreo con cercas reduce considerablemente el coste de la mano de obra y facilita su manejo, por lo que a priori podrían estudiarse acciones para aumentar su uso.

La edad media del destete (49 días) es mayor que la obtenida por García et al. (2011b) en explotaciones de ovejas Alcarreñas, con 42 días de media de lactancia natural. La mayor duración de la lactancia natural reduce la posibilidad de obtener mayor número de corderos por oveja y año, lo que podría ser un parámetro productivo a mejorar, buscando optimizar la reducción 


\section{ANÁLISIS Y DIAGNÓSTICO DE LOS SISTEMAS DE PRODUCCIÓN OVINA}

de los días de lactancia sin modificar el tamaño de la canal preferida por los consumidores. En cuanto al peso vivo de venta (22,6 kg de media), el valor es similar al obtenido por Milán y Caja (1999), en el que los corderos de raza Ripollesa se venden con una media de $23,3 \mathrm{~kg}$.

La prolificidad media de las explotaciones encuestadas (1,23 corderos/oveja) es muy similar a las obtenidas en diversos trabajos del sector ovino de varias regiones españolas, como es el caso del estudio de Gallego et al. (1993) sobre el ganado ovino de Castilla-La Mancha, con 1,3 corderos/ oveja, al igual que Núñez y Moyano (2005) en la Comarca de La Culebra (Zamora) o Mateos et al. (2005) en la raza ovina Talaverana. Sin embargo, la prolificidad media de ovinos de carne del Reino Unido, alcanza valores medios superiores a 1,41 corderos por oveja (Daza, 1999), lo que nos indica la posibilidad de conseguir una mayor selección genética de nuestra cabaña ovina que pueda mejorar este parámetro productivo que tanto influiría en la eficiencia de las explotaciones.

Según el estudio de Pardos et al. (2007), los ganaderos aragoneses obtienen un precio medio de venta de sus corderos muy superior al obtenido en este trabajo, 63,6 euros/cordero frente a 51,3 euros/cordero. Este dato se relaciona con el de ingresos brutos alcanzados donde según Pardos et al. (2006), en el año 2004, los ganaderos aragoneses, obtuvieron 120,9 euros/oveja, frente a los 83,9 euros/oveja de este estudio, dato muy similar al obtenido por Salcedo y García (2005) de los ganaderos de la provincia de Granada con unos ingresos de 86,5 euros/oveja.

Los altos ingresos conseguidos por los ganaderos aragoneses, se debe principalmente a los elevados precios de venta de los corderos registrados ese año, muchos de ellos pertenecientes a la IGP Ternasco de Aragón y por lo tanto obtienen un precio de venta mayor que el cordero convencional.

Las principales fortalezas que presenta la situación actual de los ganaderos son:

- Las razas que componen la gran mayoría del censo ovino son razas autóctonas, muy rústicas y adaptadas al medio.

- Mano de obra relativamente joven.

- Porcentaje considerable de ganaderos que de partida estarían interesados en acogerse a la IGP pudiendo ser los precursores de la expansión de la misma.

Las principales debilidades son:

- Bajo nivel genético de gran parte de la cabaña.

- Escaso fomento de la pureza racial, siendo reducido el porcentaje de ganaderos que pertenecen a ANCOS.

- Dificultad en la continuidad de las explotaciones.

- Escaso espíritu cooperativista de los ganaderos.

- Tradicional ausencia de formación profesional adecuada.

- Mano de obra escasa, relativamente cara y poco capacitada.

\section{CONCLUSIONES}

La producción ovina se desarrolla en condiciones muy frágiles, baja rentabilidad mayoritariamente en pequeños rebaños.

El relevo generacional asegurado, es escaso. Habría que potenciarlo a través de acciones que estimulen a las jóvenes generaciones a considerar atractiva la actividad.

Se detectan comarcas en las que los ingresos brutos por venta de corderos varían considerablemente, siendo en ambos casos la venta de un cordero convencional de similares características. Sería interesante realizar un análisis inferencial que pudiera explicar las causas de dichas diferencias y comprender qué aspectos son los favorables para obtener precios de venta mayores y cuáles habría que evitar.

La mayoría de los ganaderos encuestados producen cumpliendo gran parte de las exigencias que la IGP Cordero Segureño estipula, con lo que desde el punto de vista técnico y de manejo, la promoción y difu- 


\section{MARÍN-BERNAL YNAVARRO-RÍOS}

sión de este sello de calidad no supondría una dificultad importante, aspecto positivo para su implantación.

La continuidad de este estudio sería establecer análisis inferenciales que confirmen los indicios más destacables que ha señalado la estadística descriptiva, estable-

\section{BIBLIOGRAFÍA}

Avellanet, R. 2006. Conservación de recursos genéticos ovinos en la raza Xisqueta: Caracterización estructural, racial y gestión de la diversidad en programas in situ. Tesis Doctoral. Universidad Autónoma de Barcelona (UAB). $282 \mathrm{pp}$.

Castel, J.M.; Ruiz, F.A.; Mena, Y. and SánchezRodríguez, M. 2010. Present situation and future perspectives for goat production systems in Spain. Small Ruminant Res, 89: 207-210.

Círia, J.; Buxadé, C.; García, Y. y González, M.J. 1991. Estructura de las explotaciones ovinas en Soria. Actas 31. ${ }^{\text {a }}$ Reunión Científica SEEP. Tenerife. CITA-Univ. La Laguna. pp. 139-141.

Daza, A. 1999. Explotación de ovino de carne en el Reino Unido. Mundo Ganadero, 107: 40-43.

Daza, A. 2002. Mejora de la productividad y planificación de explotaciones ovinas. Ed. Agrícola española. Madrid. 232 pp.

De Rancourt, M.; Fois, N.; Lavin, P.; Tchakérian, E. y Vallerand, F. 2006. Análisis económico de las explotaciones de ganado ovino y caprino en los países de la Europa Mediterránea. XXXI Jornadas científicas. X Internacionales de la Sociedad Española de Ovinotecnia y Caprinotecnia. Zamora. 148-151.

Gallego, L.; Albiñana, B.; Torres, A.; Molina, A.; Balasch, S.; Rodríguez, M.; Fernández, N.; Díaz, J.R. y Caja, G. 1993. Caracterización de los sistemas de explotación de ganado ovino en Castilla-La Mancha. Universidad de Castilla-La Mancha. Consejería de Agricultura y Medio Ambiente. Junta de Comunidades de Castilla-La Mancha. 85 pp.

García, J.; Rouco, A. y Correal, E. 2005. Análisis económico de las explotaciones de ganado ovino y caprino en la Región de Murcia. An Vet, 21, 109-120.

García, L.; Palacios, C.; Alvarez, S. y Colmenar L. ciendo tipologías que puedan arrojar luz acerca de posibles diferencias significativas entre las distintas zonas que forman parte del área de la IGP Cordero Segureño, y plantear así acciones de asesoramiento adaptadas a las particularidades de cada territorio.

2011a. Caracterización de las explotaciones de ganado ovino de raza Alcarreña. I. Datos sociales y estructurales. AIDA. XIV Jornadas sobre Producción Animal. 41 Jornadas de Estudio. Tomo I. pp.109-111.

García, L.; Palacios, C.; Alvarez, S. y Colmenar, L. 2011b. Caracterización de las explotaciones de ganado ovino de raza Alcarreña. II. Producción y comercialización. AIDA, XIV Jornadas sobre Producción Animal. 41 Jornadas de Estudio. Tomo I. pp. 112-114.

Gaspar, P.; Escribano, A.J.; Mesías, F.J.; Escribano, M. and Pulido, A.F. 2011. Goat systems of Villuercas-lbores area in SW Spain: Problems and perspectives of traditional farming systems. Small Ruminant Res, 97: 1-11.

Mainar, R. 1995. Caracterización de la explotación de pequeños rumiantes de la C.A.M. e identificación de factores asociados con la seroprevalencia frente a diversas infecciones mediante encuesta y análisis multivariante. Tesis doctoral. Universidad Complutense de Madrid (UCM). $184 \mathrm{pp}$.

Mateos, J.J.; Cerdeño, A. y Daza, A. 2005. La raza ovina Talaverana: Estudio preliminar para su caracterización reproductiva. Actas de las XI Jornadas de Producción Animal. ITEA. Tomo I: 419-421. Zaragoza.

Milán, M.J. 1997. Las explotaciones ovinas de raza Ripollesa en Cataluña. Caracterización y establecimiento de tipologías. Tesis doctoral. Universidad Politécnica de Valencia (UPV). 290 pp.

Milán, M.J. y Caja, G. 1999. Caracterización estructural de las explotaciones ovinas de Raza Ripollesa en Catalunya. ITEA, 95 A: 91-107.

Núñez, C. and Moyano, F.J. 2005. Productive and economic parametres in semi-extensive sheep in the west of La Culebra (Zamora). Options Méditerranéennes, Série A, 70: 165-170. 


\section{ANÁLISIS Y DIAGNÓSTICO DE LOS SISTEMAS DE PRODUCCIÓN OVINA}

Pardos, L.; Buñuel, M.; Bru, C.H.; Riagues, L.; Vicente, O. y Fantova, E. 2006. Evolución de los índices técnicos y resultados económicos en explotaciones ovinas en Aragón (periodo 19952004). XXXI Jornadas científicas. X Internacionales de la Sociedad Española de Ovinotecnia y Caprinotecnia. Zamora. pp. 141-144.

Pardos, L.; Maza, M. y Fantova, E. 2007. Influencia de la prima fija en los resultados técnicos y económicos de explotaciones ovinas de carne en Aragón. ITEA, 28: 291-293.

Salcedo, A. and García, R. 2005. Sheep production systems in the north of Granada province. Case studies. Options Méditerranéennes. Série A, 70: 101-109.

Sierra, I. 2003. Evolución y cambio en el sector ovino-caprino en España durante la última dé- cada. Ed. Ministerio de Agricultura, Pesca y Alimentación. Madrid. 165 pp.

Toro-Mujica, P. 2011. Análisis técnico, económico y social del sistema ovino lechero ecológico en Castilla-La Mancha: eficiencia y sustentabilidad. Tesis doctoral. Universidad de Córdoba. 333 pp. Toro-Mujica, P.; García, A.; Gómez-Castro, A.; Perea, J.; Rodríguez-Estévez, V.; Angón, E. and Barba, C. 2012. Organic dairy sheep farms in south-central Spain: Typologies according to livestock management and economic variables. Small Ruminant Res, 104: 28-36.

Valerio, D.; García, A.; Acero, R.; Perea, J.; Tapia, M. y Romero, M. 2010. Caracterización estructural del sistema ovino-caprino de la región noroeste de República Dominicana. Arch Zootec, 59: 333-343. 